\title{
Lateral Solute Mixing Processes-A Key for Understanding Field-Scale Transport of Water and Solutes
}

\author{
H. FLÜHLER ${ }^{1}$, W. DURNER ${ }^{2}$ and M. FLURY ${ }^{3}$ \\ ${ }^{1}$ Soil Physics, Institute of Terrestrial Ecology, Swiss Federal Institute of Technology Zürich \\ ETHZ, Grabenstrasse 3, 8952 Schlieren (Switzerland) \\ ${ }^{2}$ Hydrology, University of Bayreuth, 95440 Bayreuth (Germany) \\ ${ }^{3}$ Department of Soil and Environmental Sciences, University of California at Riverside, River- \\ side, 92521 California (U.S.A.)
}

\begin{abstract}
Flühler, H., Durner, W. and Flury, M. 1995. Lateral Solute Mixing Processes-A Key for Understanding and Modeling Field-Scale Transport of Water and Solutes. Geoderma, 00: 000-000.

Lateral mass exchange mechanisms affect the spreading of solutes in the main direction of flow. Modeling vertical solute spreading requires therefore an understanding of the lateral transport across regions of varying velocities. Experimental observations show that the variable extent and rates of lateral mixing cause dramatically different transport regimes, which can neither be predicted nor explained mechanistically in terms of known state variables. In this paper we show that the various solute flow regimes are sensitive to the relative magnitudes of the vertical and lateral solute particle velocities. We discuss the concepts of describing lateral mass exchange as used in conceptually different transport models, and we postulate that physically meaningful parameters describing the lateral mixing would be a clue for deciding a priori which solute transport regime is appropriate in a particular soil or soil horizon.
\end{abstract}

\section{Introduction}

Most models used for describing the transport of water and solutes through soils wereand still are-based on the belief that the heterogeneous flow fields of water and solutes in unsaturated soils can be treated as a continuum with spatially averaged transport properties. For instances, in the Buckingham-Darcy equation (Darcy, 1856; Buckingham, 1907) any irregularities of the water flow field are lumped into the unsaturated conductivity $k(\psi)$ and soil moisture capacity $C(\psi)=d \theta / d \psi$, which are used in the mass conservation equation (Richards, 1931) and relate changes of the volumetric water content $\theta$ to those of the water potential $\psi$. Similarly, the advection-dispersion equation (solute mass conservation equation) describes the transport of solutes by means of the average velocity $v$ and a "diffusion like" spreading around the moving center of mass, defined by the velocity dependent hydrodynamic dispersion coefficient $D(v)$. The parameter functions $k(\psi), C(\psi)$, and $D(v)$ occupy a central place in the soil physics literature, but despite the continued efforts they have a poor predictive power for field-scale water and solute transport. Using these parameters in the equations mentioned above they can often be convincingly fitted to experimental data but fail when they are used for extrapolation in space and time. Model predictions are best in case of water redistribution but inconsistent with observations when the assumed equilibrium perpendicularly to the flow direction is violated. In terms of modeling success to describe correctly the distribution of one or more solute tracers is a more stringent condition than describing the 
water status or just the net water input-output balance. The trust in these equations and corresponding parameter values was founded on the outcomes of laboratory studies with packed homogeneous soil columns in which the Darcy flow and Taylor dispersion (Taylor, 1953) describe the transport regime. Systematic deviations from the expected transport behavior in less homogeneous porous materials (Coats and Smith, 1964), in undisturbed soil columns (Kissel et al., 1973; Cassel et al., 1975; van Genuchten and Wierenga, 1977) and in field soils (Wild and Babiker, 1976) was the inducement for various refinements of the advectiondispersion equation. Field-scale solute transport experiments carried out during the last ten years have shown that the Richards- and the advection-dispersion equation may be valid in some cases and fail in many others (Jury and Flühler, 1992). In unsaturated soils, erratic flow patterns classified as preferential flow, fingering, pulse splitting, or stochastic-advective as opposed to advective-dispersive transport are rather the rule than the exception. Examples of these flow pathways are given in Fig. 1, and have been documented qualitatively in numerous cases (Way, 1856; Lawes et al., 1881; Lawes et al., 1882; Bouma and Dekker, 1978; White, 1985).

Observed flow patterns are often dramatically different from what is expected from the predictions of standard solute transport models using Darcy flow and Taylor dispersion. Examples of such tracer distributions are shown in Roth et al. (1991), Ritsema and Dekker (1993), and Flury et al. (1994).

Below we distinguish three flow regimes which are summarized in Fig. 2. (We use the expression regime as a phenomenological transport feature that is dominated by one or more transport mechanisms. In the context of models we use the term process that generates the dominant system behavior. Hence, mechanisms is a physical explanation and process a model abstraction.)

1. Distribution flow in the attractor zone: Funneling of solutes into preferential pathways indicate strong flow convergence toward preferred "entry ports". Under such conditions the displacement of solutes by diffusion and hydromechanic mixing perpendicularly to the local flow direction is negligibly small. Regions of less negative water potentials or previously wetted pathways are attractors of converging streamlines.

2. Preferential flow in the transmission zone: When solutes move over considerable distances through a region of rapid flow, then the probability of their lateral escape may, for various physical reasons, be quite low. Once funnelled into a rapid flow region they travel faster than "piston" or convective transport.

3. Dispersive flow in the dispersion zone: Within a structurally isotropic and initially homogeneously wetted region the lateral flow velocities are no longer negligible but still smaller compared with those in the main transport direction. Under such conditions solutes have a higher tendency of lateral escape than in the transmission zone. Therefore they will explore neighboring flow regions of different velocities thereby loosing their memory of previously experienced velocities. Lateral dispersive mixing terminates preferential flow paths and forces the solutes into the surrounding matrix.

An important question is what system properties enhance or prevent lateral spreading or funnelling of solutes within an unsaturated or partly saturated soil horizon or profile. Despite intense efforts during the last several decades, our understanding of solute spreading remains unsatisfactory. The reason is, in our opinion, that we have concentrated too long on parameterizing lateral transport of solutes exclusively in the vertical direction. The dilemma is that soil properties important for solute transport have often spatial scales which are different in lateral and longitudinal directions. In some cases, the correlation lengths of structural properties may be of the same order as the entire transport distance through the unsaturated soil. Lumping the heterogeneity of the three-dimensional flow field into effective "vertical" 
parameters has obvious shortcomings under these spatially different length scales. It is the purpose of this paper to discuss how to improve the prediction of the vertical solute distribution by incorporating the advective and diffusive water and solute exchange in the horizontal plane. In particular, we focus on: (i) the relationship between flow regime and local velocity distribution and (ii) the model concepts of lateral water and solute exchange.

\section{Local Velocity Distribution and Flow Regime}

In this section we discuss the local velocity distribution and its influence on the macroscopically observable solute spreading in the vertical direction. We do this explicitly because these concepts are of paramount importance for field-scale transport and have not yet found sufficient attention by many model developers and model users in this field. Microscopically, the water and the dissolved particles move in all directions through the pore space. The velocity of a particle usually has three components, in $\mathrm{x}-, \mathrm{y}-$, and $\mathrm{z}$-direction. For molecular diffusion in an isotropic medium, the distribution of the particle's diffusional velocity is symmetric, but in presence of advection, the particles move preferentially in direction of the main flow. We will denote the direction parallel to the main flow as longitudinal, the direction perpendicular to it as lateral or transversal. Using the expression main direction of flow implies the definition of a specific observation scale. On a small observation scale, for instance, the main direction of flow toward a funnel neck is certainly different from the main direction of flow through the entire soil profile. We need to clarify the spatial domain we are focussing on. We will use it in a local sense, that is the main flow direction in a macroscopic flow region which can deviate from the vertical direction. In soils, the flow direction of water and particles is, in the average, predominantly vertical. Often, the three-dimensional local velocity distribution is replaced by the one-dimensional distribution of the longitudinal velocity components denoted as $f_{v}(v)$.

The local velocity distribution $f_{v}(v)$ describes the heterogeneity of the flow field on a porescale basis and cannot be measured directly. The links between these local flow properties and the observable longitudinal and lateral spreading of solutes are exemplified by a pulse applied onto the soil surface. Assume that the soil is a composite of one-dimensional flow regions of different velocities (Fig. 3). Dependent on the scale of observation such flow regions may be pores, fissures, wetted fingers, the fine porous matrix, or any region with a relatively well defined velocity. The velocity $\mathbf{v}$, being constant within a flow region, is considered to be a random variable distributed over the entire flow cross section. (Bold symbols denote random variables.) The mean and variance of this distribution $f_{v}(v)$ are denoted as $\mu_{v}$ and $\sigma_{v}^{2}$. Yet, no spatial correlation structure of the random variable $\mathbf{v}$ is assumed in this context.

The fate of a particle that enters a bundle of hypothetically parallel flow regions at the inlet end is now considered. If the flow regions are non-interacting, meaning that the boundaries of each region are impermeable for particles, an individual particle remains for its entire travel time within the region it entered. This mechanisms leads to a process which is called stochastic-advective and has often been used to analyze solute transport (e.g., Dagan and Bresler, 1979; Jury, 1982; Simmons, 1982; Shapiro and Cvetkovic, 1988). If a particle can move laterally through the boundary of its flow region, it experiences the velocities of other flow regions. This process is called advective-dispersive when the lateral mixing is fast compared to the travel time of a particle. (The widely used term convective-dispersive is synonymous in this context.) The effects of these two process assumptions on the solute concentration distribution is discussed next.

\section{Stochastic-Advective Process}

Assuming no lateral displacement, the travel distances of a single particle which entered at 
time $t=0$ into a flow region is given by

$$
\mathbf{z}=\mathbf{v} t
$$

where $\mathbf{z}$ is the vertical distance, $\mathbf{v}$ is the (vertical) velocity, and $t$ is the time. As the velocity $\mathbf{v}$ is a random variable and $t$ is constant, the particle position $\mathbf{z}$ is also a random variable. The distribution of $\mathbf{z}$ at a given time is given by (Papoulis, 1984)

$$
f(z)=f(v t)=\frac{1}{t} f_{v}\left(\frac{z}{t}\right)
$$

where $f_{v}\left(\frac{z}{t}\right)$ denotes the local velocity distribution. Equation (2) relates the distribution of travel distances to the distribution of the velocities in the flow regions. The mean and variance, $\mu_{\mathrm{sa}}$ and $\sigma_{\mathrm{sa}}^{2}$, of the distribution $f(z)$ generated by the stochastic-advective process (denoted by the subscript sa) are calculated as

$$
\begin{aligned}
\mu_{\mathrm{sa}} & =\int z f(z) d z=\int z \frac{1}{t} f_{v}\left(\frac{z}{t}\right) d z \\
& =t \int v f_{v}(v) d v \\
& =t \mu_{v} .
\end{aligned}
$$

and

$$
\begin{aligned}
\sigma_{\mathrm{sa}}^{2} & =\int z^{2} f(z) d z-\mu_{\mathrm{sa}}^{2}=\int z^{2} \frac{1}{t} f_{v}\left(\frac{z}{t}\right) d z-\mu_{\mathrm{sa}}^{2} \\
& =t^{2} \int v^{2} f_{v}(v) d v-\left(t \mu_{v}\right)^{2}=t^{2}\left(\int v^{2} f_{v}(v) d v-\mu_{v}^{2}\right) \\
& =t^{2} \sigma_{v}^{2} .
\end{aligned}
$$

where in (3) and (4) we have used the variable substitution $v=z / t$. Equations (3) and (4) relate the first two moments of the travel distance distribution to the moments of the velocity distribution. The mean of $f(z)$ increases linearly with time, and the variance increases quadratically with time. This is the characteristic of the stochastic-advective process (Simmons, 1982; Jury and Roth, 1990).

\section{Advective-Dispersive Process}

This process is based on the assumption of complete lateral mixing and requires that the transport time in the lateral direction is short relative to that in the longitudinal direction. Thus, in the advective-dispersive flow regime the velocities of an individual particle are at most weakly correlated and the particles undergo in the average all velocities due to lateral movement. The travel distance of a particle at a given time can be calculated as the sum of many small travel distances within $n$ different flow regions:

$$
\mathbf{z}=\mathbf{z}_{1}+\cdots+\mathbf{z}_{n}
$$

The incremental travel distances $\mathbf{z}_{i}$ are in this case independent random variables. The central limit theorem states that for sufficiently large $n$ the distribution of their sum, that is of $\mathbf{z}$, tends to a normal distribution. Thus, irrespective of the local velocity distribution the travel distances approximate a normal distribution.

We denote the time spent by a particle within a particular flow regions by $\Delta t$. Then we can write Equation (5) as

$$
\begin{aligned}
\mathbf{z} & =\mathbf{v}_{1} \Delta t+\cdots+\mathbf{v}_{n} \Delta t \\
& =\left(\mathbf{v}_{1}+\cdots+\mathbf{v}_{n}\right) \Delta t
\end{aligned}
$$


where $n$ is determined by $n=t / \Delta t$. As the $\mathbf{v}_{i} \mathrm{~s}$ are independent, identically distributed random variables, we can calculate the mean and variance of the sum $\mathbf{z}$ by (Papoulis, 1984)

$$
\begin{gathered}
\mu_{\mathrm{ad}}=n \mu_{v} \Delta t=t \mu_{v} \\
\sigma_{\mathrm{ad}}^{2}=n \sigma_{v}^{2} \Delta t=t \sigma_{v}^{2}
\end{gathered}
$$

where the subscript ad denotes advective-dispersive. Equations (7) and (8) are analogous to (3) and (4). In the advective-dispersive process the mean and variance of the travel distances grow linearly with time.

\section{Illustration}

The distribution of the travel distance $\mathbf{z}$, denoted as $f(z)$, corresponds to the distribution of resident concentrations after a Dirac pulse application of unit mass. The first two moments, Eqs. (3) and (4) for the stochastic-advective process, and (7) and (8) for the advectivedispersive process denote the center of mass and the spreading of concentrations along the transport direction.

Figure 4 illustrates the effect of the local velocity distribution and process assumption on the distribution of the travel distances. We assume a local velocity distribution that is bimodal log-normal (top of Fig. 4)

$$
f_{v}(v)=\sum_{i=1}^{2} \omega_{i} \frac{1}{v \sigma_{i} \sqrt{2 \pi}} \exp \left(-\frac{\left(\log (v)-\mu_{i}\right)^{2}}{2 \sigma_{i}^{2}}\right),
$$

where $\mu_{i}$ and $\sigma_{i}$ are the parameters of the log-normal distribution, not to be confounded with its moments, and $\omega_{i}$ is a weighting factor that satisfies $\omega_{1}+\omega_{2}=1$. The parameters and weighting factors in (9) were chosen such that $f(z)$ contains a small fraction of large velocities. We use this hypothetical velocity distribution to show that its shape is irrelevant in case of a dispersive flow regime but a dominant property in case of a (stochastic-)advective preferential flow regime. The distributions of the travel distances $f(z)$, which can be considered as a normalized resident concentration distributions with depth, were calculated with (2) for the stochastic-advective process and with (7) and (8) for the advective-dispersive process. This travel distance probability density functions of the two processes are shown in Fig. 4 for two different times, where $t_{1}<t_{2}$. Two aspects are worth noting. Firstly, the shapes of $f(z)$ look different. In absence of lateral mixing (stochastic-advective flow regime) the shape of the local velocity distribution is reproduced in the distribution of travel distances. The bimodal velocity distribution results in a bimodal distribution of travel distances (Fig. 4, left column). For complete (advective-dispersive) mixing the bimodal velocity distribution leads to a unimodal distribution of the travel distances (Fig. 4, right column). Secondly, Fig. 4 illustrates the behavior of the variance of $f(z)$ for the two processes at different times $t_{1}$ and $t_{2}$ (Eqs. (4) and (8)). The variance increases much faster with time for the stochasticadvective than for the advective-dispersive process. The two flow regimes represent extremes in lateral mixing behavior. In real soils, we expect to observe transitions between these two limiting cases. It is important to realize that in some cases the velocity distribution leaves its trace in occasionally "erratic" concentration distributions, for instance in cases when a single solute pulse is separated in two or several pulses. When we use a particular local velocity distribution to generate advective transport in stochastically distributed isolated flow regions, it is possible to fit $f_{v}(v)$ to field data of such transition cases which may neither be purely stochastic-advective nor purely advective-dispersive. Doing this, the shape of $f_{v}(v)$ is merely a fitting option to account for the observed degree of lateral and longitudinal mixing, similar to that of defining an apparent dispersion coefficient. 


\section{Concepts for Modeling Lateral Mixing}

The three-dimensionality of solute flow can be conceptualized in rather different ways. Due to the highly irregular spatial array of transport-relevant soil properties, the use of a three-dimensional deterministic modeling approach is generally not feasible. Below we describe examples of parameterizing the lateral transport of conservative tracers. Conceptual differences for describing the mixing of solutes between the flow regions that discriminate preferential from dispersive flow are emphasized.

\section{Mass Exchange between Two Discrete Regions}

As discussed earlier, the simple (one-region) version of the advection-dispersion model has been modified to account for the systematic deviations between observed and calculated breakthrough curves or, less frequently, also for fitting depth profiles of tracer concentrations.

The approach most frequently used to describe preferential flow consists of partitioning the soil into two subdomains, one which is used for transport, and another, which represents a storage capacity. These so called dual-porosity, two-region or bi-continuum models have been extensively studied in such wide ranging fields as fissured groundwater and petroleum reservoirs (Barenblatt et al., 1960; Warren and Root, 1963; Coats and Smith, 1964), in flow and transport in saturated and unsaturated rock (Wang and Narasimhan, 1985; Pruess et al., 1990), and soils (van Genuchten and Wierenga, 1976). For numerous column experiments the parameters of the two-region model have been estimated with the fitting-routine of Parker and van Genuchten (1984). As Gerke and van Genuchten (1993) pointed out, that the dualporosity model includes as special cases (i) compartment or first-order rate models in which advective transport in the region of low permeability is neglected (immobile region), (ii) fissured medium models where the storage term for the mobile region is assumed to be negligible, and (iii) microstructure models where the dynamics of flow and transport is considered at the local scale. The various classes of such models were discussed by Berkowitz et al. (1988) and Piquemal (1993).

For any two-region model the exchange of fluid and solutes between the two pore regions must be specified. Brusseau and Rao (1990) listed three basic mechanisms for solute exchange: (i) the coupling of the two regions is instantaneous, which results in a single equivalent porous medium where the effect of the immobile phase is lumped into an effective dispersion coefficient (Passioura, 1971); (ii) the mass exchange is treated as a diffusion-like process driven by an apparent concentration gradient between the mobile $(\mathrm{m})$ and immobile $(\mathrm{im})$ region and controlled by a diffusional resistance or mass transfer coefficient $\alpha$

$$
\theta_{i m} \frac{\partial c_{i m}}{\partial t}=\alpha\left(c_{m}-c_{i m}\right)
$$

or (iii) the physical process of mass exchange is modeled explicitly. The latter requires knowledge of the transport mechanisms from the bulk solution to the mobile-immobile boundary layer, across the interface, and within the immobile region. In addition, the explicit mechanistic approach requires a medium with a well-defined geometry and the knowledge of a usually not well defined apparent diffusion coefficient within the immobile region (Rasmuson and Neretnieks, 1980; Rao et al., 1980a; Rao et al., 1980b; Lafolie and Hayot, 1993). Under field conditions, this detailed information is rarely available.

Raats (1981) showed that the first two of the above mentioned model types, (i) and (ii), are degenerate cases of the physical diffusion model. van Genuchten (1985) and van Genuchten and Dalton (1986), among others, compared the different models and found relations between the physical and the lumped parameters. Apparent diffusion into and out of porous spheres of radius $r$ and porosity $\left(\theta-\theta_{m}\right)$ representing a quasi stagnant, immobile phase embedded in a moving fluid phase mimicks a quadratic velocity dependence of the total longitudinal 
dispersion coefficient $D_{l}$ (Parker and Valocchi, 1986)

$$
D_{l}=\frac{\theta_{m}}{\theta} D_{m}+\left[\frac{\left(1-\frac{\theta_{m}}{\theta}\right) r^{2}}{15 D_{e}}\right] v^{2},
$$

where $\theta_{m} / \theta$ is the ratio between the water content inbetween the saturated porous spheres (inter-aggregate pore space) and total water content, $v$ the average pore water velocity, $D_{e}$ the molecular diffusion coefficient of solute in stagnant water, $D_{m}$ the dispersion coefficient of the mobile phase only (as obtained with solid spheres of equal diameter), and $D_{l}$ the dispersion coefficient obtained by fitting the breakthrough curve with the simple advectiondispersion equation, that is by treating the porous spheres and the mobile phase inbetween as homogeneous porous media. The experimentally verified quadratic relationship of $D_{l}(v)$ is an example of how a mathematical formalism used for describing the lateral mass transfer is lumped into the coefficient $D_{l}$, which describes the solute spreading in the longitudinal direction (Koch and Flühler, 1993).

In contrast to the mobile-immobile model approach, other models assume transport in the main flow direction to take place in both, the high and the low permeability region at different velocities. Skopp et al. (1981) have solved the advection-dispersion equation analytically for two interacting flow regions, each characterized by its own parameter set. The two regions were coupled by a mass exchange mechanism according to Eq. (10).

Although contributing to theoretical understanding, the practical usefulness of the analytical solution is limited by the inherent steady-state assumption and the simplified initial and boundary conditions (Jarvis et al., 1991). Numerical double-porosity models for variably saturated water flow and solute transport in fractured rock were proposed by Dykhuizen (1987) and Dudley et al. (1988). In the one-dimensional, numerical, non-stationary water and solute transport model by Gerke and van Genuchten (1993) water and solutes are transported in parallel through two differently mobile regions. The lateral solute exchange between the two regions is both advective and diffusive-dispersive to allow wetting and draining of the two regions at different rates. Similar to the mobile-immobile models, the solute exchange is described with a semi-empirical first-order rate equation (Gerke and van Genuchten, 1993)

$$
\Gamma_{s}=(1-d) \Gamma_{w} \phi_{m a} c_{m a}+d \Gamma_{w} \phi_{m i} c_{m i}+\alpha\left(1-w_{m a}\right) \theta_{m i}\left(c_{m a}-c_{m i}\right),
$$

where the subscripts $m i$ and $m a$ indicate the region containing the micropores and the larger pores, respectively, $\Gamma_{s}$ is the solute exchange term, defined as mass of solutes per unit volume of bulk soil and per unit time, $\Gamma_{w}$ is the water flux exchange rate between the two regions, $d$ is a direction-dependent switch (being 0 or 1), $\alpha$ is the solute transfer coefficient, $\phi_{m a}$ and $\phi_{m i}$ are dimensionless coefficients that consider the relative solute capacitance of the two regions, and $\left(1-w_{m a}\right) \theta_{m i}$ is the volume of water in the matrix pore system per unit bulk volume.

A limiting case of the two-region model category are the macropore models where the soil is divided into a small macropore region and a large microporous matrix region (Jarvis et al., 1991; Chen and Wagenet, 1992). The non-stationary water and solute transport through the macropores is described by various simplified concepts, for instance the kinematic wave approach or the Chezy-Manning equations for tube flow, whereas the transport in the matrix is described by the Richards equation and is regarded as advective-dispersive. In the approach of Jarvis et al. (1991), the lateral exchange takes part by a advective flux from the macropore region (subscript $m a$ ) into the micropore region (subscript $m i$ ) and a diffusional mass transfer, which is driven again by a first-order rate expression. The latter is assumed to be proportional to the diffusion coefficient of the solute in water, $D_{o}$, and a lumped mass transfer coefficient $\alpha$ (Jarvis et al., 1991)

$$
\theta_{m i} \frac{\partial c_{m i}}{\partial t}=\alpha D_{o}\left(c_{m a}-c_{m i}\right) \theta_{m a}+q_{w} c_{m a}
$$


Chen and Wagenet (1992) restrict the lateral solute exchange to purely advective transport from the macropore to the micropore region. The horizontal water exchange is described by an infiltration equation.

Bi-continuum models with two differently mobile regions that consider water and solute exchange between the regions are very parameter demanding. This restricts their practicability for field situations. However, it appears that preferential flow patterns are often associated with non-stationary water transport both laterally as well as vertically (Ritsema and Dekker, 1993). Such non-stationarities would justify the additional complexity and parameter requirements.

\section{Multiple Flow Regions without Mass Exchange}

The separation of a porous medium into two flow regions has physical limitations. Each flow region contains a wide distribution of pore-sizes and is therefore a composite of many differently mobile regions. Further, dual-porosity models cannot be fitted to experimental breakthrough curves exhibiting extensive tailing (Brusseau and Rao, 1990). Moreover, the mobile-immobile model used for undisturbed soil columns give model parameters that vary with water content (Zurmühl, 1994). Multiregion models overcome some of the shortcomings of dual-porosity models.

Lindstrom and Boersma (1971) proposed an analytical multi-region model where lateral exchange between the regions was neglected. Transport in each region was described by an independent advection-dispersion process with a pore-size specific mean transport velocity resulting in very early solute breakthrough and extensive tailing, an occurrence which is seldom observed in soils (Rao et al., 1976).

Stochastic-advective transport models can be regarded as special cases of multiple region models with noninteracting regions (e.g., Dagan and Bresler, 1979; Jury, 1982; Simmons, 1982; Shapiro and Cvetkovic, 1988). Although lateral mass exchange is neglected, longitudinal spreading of a pulse is achieved by choosing an appropriate shape of the velocity distribution as shown in Fig. 4. As pointed out earlier, this velocity distribution is not equal to the local velocity distribution of water flow. Therefore an (arbitrary) type of transport velocity distribution function is assumed and its parameters determined by fitting to the data of a transport experiment. A log-normal velocity distribution, for instance, produces a leading tail in the main direction of flow. As shown in the section on the stochastic-advective process, models of this type predict a quadratic increase in the travel time variance with travel distance.

\section{Multiple Flow Regions with Lateral Mass Exchange}

The two flow regions of dual-porosity models completely border each other whereas the distinction of multiple regions requires a measure of "neighborhood" between the regions. Typically, the pore classes are arranged on a one-dimensional axis perpendicularly to the direction of flow. This coordinate, $\xi$, represents either pore sizes (Durner and Flühler, this issue) or a measure of spatial distance between pore regions (see Roth et al. (1990) and Fig. 2 in Skopp and Gardner (1992) for illustrations of this concept).

For the treatment of lateral mass exchange between the regions, two extreme cases can be distinguished: an equal interaction between all pore classes (Steenhuis et al., 1990) and an interaction that is most intense for neighboring classes (Skopp and Gardner, 1992; Durner and Flühler, this issue)). The flow resistance for solute exchange between the regions must be specified in such models. Extreme cases are equal interaction between all pore classes (Steenhuis et al., 1990) or an interaction which defines for each pore class one or two neighborhood classes (Skopp and Gardner, 1992; Roth et al., 1990; Durner and Flühler, this issue). A flexible way to model this is to introduce an additional structural non-cartesian coordinate, which can represent pore sizes, velocities, or a measure of spatial distance between pore regions. 
Skopp and Gardner (1992) investigated analytically the dependence of the longitudinal dispersion coefficient on the pore-water velocity distribution in a saturated porous medium. They started out from a differentiable velocity distribution $v(\xi)$ of arbitrary shape. Lateral mass exchange between the multiple flow regions was treated as a diffusion-like process in the direction of a velocity-coordinate $\xi$, where $\xi$ is a dimensionless parameter with values $0 \leq \xi \leq 1$. The mass transfer $J_{\xi}$ between regions of similar velocities is given as (Skopp and Gardner, 1992)

$$
J_{\xi}=L \delta(\xi) \frac{\partial c}{\partial \xi}
$$

where $L$ is a phenomenological interaction coefficient, $c$ is the solute concentration, and $\delta(\xi)$ is the pore size distribution expressed on the basis of the pore class velocities $\xi$. Basically, $L$ is a fitting parameter, which lumps the stochastic distribution of lateral average distances of pore clusters with different flow velocities into one single number. Depending on $L$ this analysis yields a functional dependence of $D$ on the average velocity. Their model is consistent with the observation that $D$ increases with increasing heterogeneities in the flow field.

In the particle tracking model by Roth et al. (1990) the lateral transfer of a particle $i$ is represented as a diffusion process on a structural coordinate $\xi$ linking the flow regions of differing longitudinal average velocities

$$
\begin{aligned}
& \xi_{i}\left(t_{k+1}\right)=\xi_{i}\left(t_{k}\right)+\omega \sqrt{6 D_{\xi} \Delta t} \\
& z_{i}\left(t_{k+1}\right)=z_{i}\left(t_{k}\right)+v_{i} \Delta t+\omega \sqrt{6 D_{z} \Delta t}
\end{aligned}
$$

where $D_{\xi}$ and $D_{z}$ are the apparent diffusion and dispersion coefficient in the direction of the $\xi$ - and $z$-coordinate, respectively, and $\xi_{i}\left(t_{k}\right)$ and $z_{i}\left(t_{k}\right)$ the position of particle $i$, both defined in the structure space $(\xi, z) ; \omega$ is a uniformly distributed random number in the interval $[-1,1] ; v_{i}$ the velocity in the direction of flow; and $\Delta t$ is the time increment in the particle tracking. In this model the transport domain is divided in only two flow regions, but the number of flow regions could be adapted to any discrete velocity distribution and layer sequence. The depth distribution of the resident concentration is obtained by integrating the number of particles over the structural coordinate. Extreme flow patterns such as those observed in the soils Bülach (Roth et al., 1991) and Wülflingen (Fig. 1) can be generated by adjusting the lateral diffusion coefficient $D_{\xi}$ and the relative proportions of the flow regions of different longitudinal $v_{i}$ and $D_{z}$.

Steenhuis et al. (1990) developed a mixing cell model where the soil is subdivided into multiple flow regions that represent different pore-size classes. The sizes of the pore-classes and the water flow velocities within them depend on the unsaturated conductivity function. Within each region purely advective transport takes place assuming unit gradient conditions. The model has been solved numerically for the simplified case of steady-state water flow under unit-gradient conditions. Mass transfer between the pore regions is simulated after each time step by a discrete mixing step. At each time step and for each cell, a certain fraction $\tau_{i}$ of the soil solution of pore class $i$ is moved into a common pool, where it is mixed and replaced. The solute content $s_{i}$ is changed to $s_{i}^{\prime}$ (Steenhuis et al., 1990)

$$
s_{i}^{\prime}=s_{i}\left(1-\tau_{i}\right)+\tau_{i}^{\prime} \frac{\sum_{j=0}^{n} \tau_{j} s_{j}}{\sum_{j=0}^{n} \tau_{j}^{\prime}},
$$

where the subscripts indicate pore classes, the prime indicates the time after the mixing has taken place, $\tau_{i}$ is the fraction of fluid taken out of pore group $i$, and $\tau_{i}^{\prime}$ is the fraction that is moved back.

Durner and Flühler (this issue) generalized this concept by subdividing the soil into an arbitrarily large number of pore classes. The pore classes are arranged according to their pore sizes along a one-dimensional coordinate perpendicular to the mean direction of flow. 
In each pore class the longitudinal transport is purely advective. Lateral mass exchange between the pore classes may take place by convective transfer from large to small pores and by a diffusion-like process along this coordinate (Durner and Flühler, this issue, Eq. 10). As in the model of Skopp and Gardner (1992), the lateral separation distances between the different pore classes and the diffusional properties have to be lumped into empirical parameters. The model has been solved numerically for the simplified case of steady-state water flow under unit-gradient conditions. Simulations show how the initially stochastic-advective transport process gradually changes to an advective-dispersive one. As expected, the longitudinal dispersion largely depends on the lateral mass exchange (Fig. 5 in Durner and Flühler, this issue). Soils with bimodal pore-size distributions show pulse splitting and preferential flow when saturated, and an advective-dispersive regime at smaller water contents.

The pore-size and velocity-distribution based models quoted above have in common that a statistical assemble of flow regions is arranged on a lateral coordinate which implies a spatial correlation between regions of similar velocities or pore sizes. Lateral velocity and distance are both packed into the interaction parameters. To put numbers to this lateral transmission property we either need a broad and detailed experimental data base or more information on the spatial arrangement (correlation lengths of pertinent structures) and also the lateral permeabilities of the individual flow regions.

In contrast to the two-region models the interacting flow region models can produce almost any shape of breakthrough curves or depth distributions of solute concentration. This high degree of flexibility is achieved at the expense of a parameter identification problem. At present, these models are elegant intellectual exercises that pinpoint the lack of our knowledge and process understanding.

\section{Predicting Lateral and Longitudinal Dispersion}

With the exception of the stochastic-advective concept all of the models quoted above assume that solutes are spread laterally as they advance in the main direction of flow. A number of different two- and three-dimensional water and solute transport models explicitly model advection and dispersion in different spatial directions. For saturated horizontal transport in aquifers the data base of directional dispersivities is appreciable (Gelhar et al., 1992) but, to our knowledge, no equivalent data are available for vertical, partially unsaturated transport through soils.

Assuming that pore-scale dispersion is negligible the spatial correlation of hydraulic properties captures the influence of structural heterogeneities and provides a basis for calculating the dispersion in all directions of space (Dagan, 1984; Dagan, 1987). Dagan unravelled the contradictions between solute dispersion observed in aquifers and expected based on the Taylor dispersion assumption. He showed that at a local scale (i.e., horizon, single well, soil profile, or soil plot) or at a regional scale (aquifer) the spatial array of hydraulic conductivities $(Y=\log k)$ and, correspondingly, of the three-dimensional distribution of flow regions and velocities, explain the spreading of a solute plume in all directions. The directional spatial correlation of $Y$ is expressed as integral scale $I_{r}$. This correlation length $I_{r}$ is calculated as the area under the autocorrelation function $\rho_{Y}(r)$ of $Y\left(\int_{0}^{\infty} \rho_{Y}(r) d r\right)$ in the direction $\vec{r}$. The ratio between $I_{t} / I_{l}$ in the lateral $(t)$ and longitudinal $(l)$ direction determines the nature of the flow regime. The subscript $t$ stands for transverse. Since the hydraulic conductivity is a macroscopic variable averaged over a region much larger than the pore scale, the predicted mixing is of a purely advective nature. A large longitudinal and small lateral correlation favors the formation of preferential flow.

\section{Generating Spatial Flow Patterns}

Under unsaturated conditions with varying water contents the situation might be more complex. In a numerical analysis of solute transport in unsaturated soil, Russo et al. (1994a) 
found a decrease in overall longitudinal dispersion with increasing average water content, which was attributed to higher heterogeneity of the soil properties at lower water content. In the same study, (Russo et al., 1994a,b) the key role of lateral exchange for the longitudinal spreading was confirmed numerically by comparing the transport behavior under intermittent irrigation and under a uniform pressure head boundary. Under uniform boundary conditions the lateral water movement and consequently the lateral mixing was small, whereas the longitudinal dispersion was high. Under pulsing irrigation, the redistribution of water lead to an increased lateral mixing, which resulted in a smaller longitudinal dispersion. Layering may have opposite effects under wet and desaturated conditions (Kung, 1990a,b). Even under a regime of a continuous unsaturated infiltration the non-stationarities of the water flow field may persist because the slightest pulsing of irrigation or rainfall and the spatially variable permeabilities near or at the surface propagate and enhance the hydraulic non-equilibria. In contrast to flow in extended aquifers, diffusion or pore scale mixing might be relevant for the flow regime in presence of less permeable soil structures.

Flury (1993) has shown that the extremely different flow patterns observed in field soils can be generated by means of the diffusion-limited aggregation algorithm. Particles are displaced from the bottom of the profile with a random walk process until they reach the surface of the profile or a location where another particle is already residing. This random-walk is defined by four probabilities, two for the lateral and two for the vertical directions. This process does not describe the fate of a moving particle, as for instance in the particle tracking model by Roth et al. (1990), but the result of the process describes the spatial structure of the flow paths. From this analysis it seems that a flow pattern within a given horizon does not change in the course of the infiltration event. For this reason it is possible to calibrate the DLA-model parameters for a given time or depth and to extrapolate the concentration distribution to future times or depths.

\section{Conclusions}

If we knew the horizontal flux of water and matter in soils we could very likely (re)predict even the most erratic flow patterns observed under field conditions. A fairly broad selection of models is available to describe or rather to reproduce analytically or numerically both, the transport in the longitudinal direction as well as the exchange between regions of different mobility. These models have in common that the lateral exchange can be estimated at best by fitting model solutions to breakthrough curves, to depth distributions of resident concentrations, or to two- (or rarely three-) dimensional tracer distributions. However, available experimental data for model calibration are scarce and insufficient for model validation. There is a large demand for experiments, both in the field and laboratory, to explore the causes and extent of lateral solute movement. Especially, the reasons of preferential flow initiation should be investigated, that is the separation of solutes within the horizon overlaying the transmission zone. It is probable that different causes produce similar patterns. Hence a particular flow pattern might be the result of different initial conditions, spatially variable hydraulic properties, structural discontinuities or wetting patterns imposed at the soil surface, among others. It is conceivable that we need more than just the first two moments of observed travel times or of depth distributions to capture the characteristics of a specific transport regime.

\section{Acknowledgment}

W. Durner thanks the European Environmental Research Organization (EERO) for providing support for a long-term fellowship. 


\section{References}

Barenblatt, G. I., Zheltov, I. P. and Kochina, I., 1960. Basic concepts in the theory of seepage of homogeneous liquids in fissured rocks. J. Appl. Math. Mech., 24: 1286-1303.

Berkowitz, B., Baer, J. and Braester, C., 1988. Continuum models for contaminant transport in fractured porous formations. Water Resour. Res., 24(8): 1225-1236.

Bouma, J. and Dekker, L. W., 1978. A case study on infiltration into dry clay soil. I. Morphological observations. Geoderma, 20: 27-40.

Brusseau, M. L. and Rao, P. S. C., 1990. Modeling solute transport in structured soils: A review. Geoderma, 46: 169-192.

Buckingham, E., 1907. Studies on the movement of soil moisture. U.S. Department of Agriculture Bureau of Soils, Government Printing Office, Bulletin 38, Washington D.C.

Cassel, D. K., van Genuchten, M. T. and Wierenga, P. J., 1975. Predicting anion movement in disturbed and undisturbed soils. Soil Sci. Soc. Am. J., 39: 1015-1019.

Chen, C. and Wagenet, R. J., 1992. Simulation of water and chemicals in macropore soils. Part 1. Representation of the equivalent macropore influence and its effect on soilwater flow. J. Hydrol., 130: 105-126.

Coats, K. H. and Smith, B. D., 1964. Dead-end pore volume and dispersion in porous media. Soc. Petr. Eng. J., 4: 73-84.

Dagan, G., 1984. Solute transport in heterogeneous porous formations. J. Fluid Mech., 145: 151-177.

Dagan, G., 1987. Statistical theory of groundwater flow and transport: Pore to laboratory, laboratory to formation and formation to regional scale. Ann. Rev. Fluid Mech., 19: 183215.

Dagan, G. and Bresler, E., 1979. Solute dispersion in unsaturated heterogeneous soil at field scale, I, Theory. Soil Sci. Soc. Am. J., 43: 461-467.

Darcy, H., 1856. Les fontaines publiques de la ville de Dijon. Dalmont, Paris.

Dudley, A., Peters, R. R., Gauthier, J. H., Wilson, M. L., Tierney, M. S. and Klavetter, E. A., 1988. Yucca Mountain Project, total system performance assessment code (TOSPAC), vol. 1, Physical and mathematical bases. Sandia Rep. Sand85-0002. Sandia Natl. Lab., Alberquerque, N.M.

Durner, W. and Flühler, H., this issue. Multi-domain models for pore-size dependent transport of solutes in soils. Geoderma.

Dykhuizen, R. C., 1987. Transport of solutes through the unsaturated zone. Water Resour. Res., 21(12): 1531-1539.

Flury, M., 1993. Transport of bromide and chloride in a sandy and a loamy soil. Ph.D. Diss. No. 10185. ETH Zürich.

Flury, M., Flühler, H., Jury, W. A. and Leuenberger, J., 1994. Susceptibility of soils to preferential flow of water: A field study. Water Resour. Res., 30(7): 1945-1954. 
Gelhar, L. W., Welty, C. and Rehfeldt, K. R., 1992. A critical review of data on field-scale dispersion in aquifers. Water Resour. Res., 28(7): 1955-1974.

Gerke, H. H. and van Genuchten, M. T., 1993. A dual-porosity model for simulating the preferential movement of water and solutes in structured porous media. Water Resour. Res., 29(2): 305-319.

Jarvis, N., Jansson, P.-E., Dik, P. and Messing, I., 1991. Modelling water and solute transport in macroporous soil. I. Model description and sensitivity analysis. J. Soil Sci., 42: 5970.

Jury, W. A., 1982. Simulation of solute transport using a transfer function model. Water Resour. Res., 18(2): 363-368.

Jury, W. A. and Flühler, H., 1992. Transport of chemicals through soil: Mechanisms, models, and field applications. Adv. Agronomy, 47: 141-201.

Jury, W. A. and Roth, K., 1990. Transfer functions and solute movement through soil. Theory and applications. Birkhäuser, Basel.

Kissel, D. E., Richtie, J. T. and Burnett, E., 1973. Chloride movement in undisturbed swelling clay soil. Soil Sci. Soc. Am. Proc., 37: 21-24.

Koch, S. and Flühler, H., 1993. Non-reactive solute transport with micropore diffusion in aggregated porous media determined by a flow-interruption method. J. Contaminant Hydrol., 14: 39-54.

Kung, K.-J. S., 1990a. Preferential flow in a sandy vadose zone: 1. Field observation. Geoderma, 46: 51-58.

Kung, K.-J. S., 1990b. Preferential flow in a sandy vadose zone: 2. Mechanism and implications. Geoderma, 46: 59-71.

Lafolie, F. and Hayot, C., 1993. One-dimensional solute transport modelling in aggregated porous media. Part 1. Model description and numerical solution. J. Hydrol., 143: 63-83.

Lawes, J. B., Gilbert, J. H. and Warington, R., 1881. On the amount and composition of the rain and drainage-waters collected at Rothamsted, Part I and II. J. Royal Agric. Soc. of England, London, 17: 241-279.

Lawes, J. B., Gilbert, J. H. and Warington, R., 1882. On the amount and composition of the rain and drainage-waters collected at Rothamsted, Part III. J. Royal Agric. Soc. of England, London, 18: 1-71.

Lindstrom, F. T. and Boersma, L., 1971. A theory on the mass transport of previously distributed chemicals in a water saturated sorbing porous medium. Soil Sci., 111(3): 192199.

Papoulis, A., 1984. Probability, Random Variables, and Stochastic Processes. 2nd edn. McGraw-Hill, Singapore.

Parker, J. C. and Valocchi, A. J., 1986. Constraints on the validity of equilibrium and firstorder kinetic transport models in structered soils. Water Resour. Res., 22: 399-407.

Parker, J. C. and van Genuchten, M. T., 1984. Determining transport parameters from laboratory and field tracer experiments. Virginia Agricultural Experiment Station. Blacksburg VA. 
Passioura, J. B., 1971. Hydrodynamic dispersion in aggregated media I. Theory. Soil Sci., 111: 339-344.

Piquemal, J., 1993. On the modelling conditions of mass transfer in porous media presenting capacitance effects by a dispersion-convection equation for the mobile fluid and a diffusion equation for the stagnant fluid. Transport in Porous Media, 10: 271-283.

Pruess, K., Wang, J. S. Y. and Tsang, Y. W., 1990. On thermohydraulic conditions near high-level nuclear wastes emplaced in partially saturated fractured tuff, 2, Effective continuum approximation. Water Resour. Res., 26: 1249-1261.

Raats, P. A. C., 1981. Transport in structured media. Proc. Euromech, 143, 1-4, September 1981, Delft: 221-226.

Rao, P. S. C., Green, R. E., Ahuja, L. R. and Davidson, J., 1976. Evaluation of a capillary bundle model for describing solute dispersion in aggregated soils. Soil Sci. Soc. Am. J., 40: $815-820$.

Rao, P. S. C., Jessup, R. E., Rolston, D. E., Davidson, J. M. and Kilcrease, D. P., 1980a. Experimental and mathematical description of nonadsorbed solute transfer by diffusion in spherical aggregates. Soil Sci. Soc. Am. J., 44: 684-688.

Rao, P. S. C., Rolston, D. E., Jessup, R. E. and Davidson, J. M., 1980b. Solute transport in aggregated porous media: Theoretical and experimental evaluation. Soil Sci. Soc. Am. J., 44: 1139-1146.

Rasmuson, A. and Neretnieks, I., 1980. Exact solution of a model for diffusion in particles and longitudinal dispersion in packed beds. Am. Ind. Chem. Eng. J., 26(4): 686-690.

Richards, L. A., 1931. Capillary conduction of liquids through porous mediums. Physics, 1: 318-333.

Ritsema, C. J. and Dekker, L. W., 1993. Soil moisture and dry bulk density patterns in bare dune sands. J. Hydrol., 154: 107-131.

Roth, K., Flühler, H. and Attinger, W., 1990. Transport of a conservative tracer under field conditions: Qualitative modelling with random walk in a double porous medium. In: K. Roth, H. Flühler, W. A. Jury and J. C. Parker (Editors), Field-Scale Water and Solute Flux in Soils, Proceedings of the Centro Stefano Franscini, Monte Verità, Ascona. Birkhäuser, Basel, pp. 239-249.

Roth, K., Jury, W. A., Flühler, H. and Attinger, W., 1991. Transport of chloride through an unsaturated field soil. Water Resour. Res., 27(10): 2533-2541.

Russo, D., Zaidel, J. and Laufer, A., 1994a. Stochastic analysis of solute transport in partially saturated heterogeneous soil. I. Numerical experiments. Water Resour. Res., 30(3): 769779.

Russo, D., Zaidel, J. and Laufer, A., 1994b. Stochastic analysis of solute transport in partially saturated heterogeneous soil. II. Prediction of solute spreading and breakthrough. Water Resour. Res., 30(3): 781-790.

Shapiro, A. M. and Cvetkovic, V. D., 1988. Stochastic analysis of solute arrival time in heterogeneous porous media. Water Resour. Res., 24: 1711-1718.

Simmons, C. S., 1982. A stochastic-convective transport representation of dispersion in onedimensional porous media systems. Water Resour. Res., 18(4): 1193-1214. 
Skopp, J. and Gardner, W. R., 1992. Miscible displacement: An interacting flow region model. Soil Sci. Soc. Am. J., 56: 1680-1686.

Skopp, J., Gardner, W. R. and Tyler, E. J., 1981. Solute movement in structured soils: Tworegion model with small interaction. Soil Sci. Soc. Am. J., 45(00): 837-842.

Steenhuis, T. S., Parlange, J.-Y. and Andreini, M. S., 1990. A numerical model for preferential solute movement in structured soils. Geoderma, 46: 193-208.

Taylor, G. I., 1953. Dispersion of soluble matter in solvent flowing through a tube. Proc. R. Soc. Series A, 219: 186-203.

van Genuchten, M. and Wierenga, P., 1977. Mass transfer studies in sorbing porous media. II. Experimental evaluation with tritium $\left({ }^{3} \mathrm{H}_{2} \mathrm{O}\right)$. Soil Sci. Soc. Am. J., 41: 272-278.

van Genuchten, M. T., 1985. A general approach for modeling solute transport in structured soil. Hydrogeology of Rocks of Low Permeability. Proc. 17th Internatl. Congr., Internatl. Association of Hydrogeologists, pp. 513-524.

van Genuchten, M. T. and Dalton, F. N., 1986. Models for simulating salt movement in aggregated field soils. Geoderma, 38: 165-183.

van Genuchten, M. T. and Wierenga, P. J., 1976. Mass transfer studies in sorbing porous media. I. Analytical solutions. Soil Sci. Soc. Am. J., 40: 473-480.

Wang, J. S. Y. and Narasimhan, T., 1985. Hydrologic mechanisms governing fluid flow in a partially saturated, fractured, porous medium. Water Resour. Res., 21: 1861-1874.

Warren, J. E. and Root, P. J., 1963. The behavior of naturally fractured reservoirs. Soc. Petr. Eng. J., 3: 245-255.

Way, T., 1856. On the composition of land-drainage and of rain. J. Royal Agric. Soc. of England, London, 7: 123-162.

White, R. E., 1985. The influence of macropores on the transport of dissolved and suspended matter through soil. Adv. Soil Sci., 3: 95-120.

Wild, A. and Babiker, L. A., 1976. The asymmetric leaching pattern of nitrate and and chloride in a loamy sand under field conditions. J. Soil Sci., 27: 460-466.

Zurmühl, T., 1994. Validierung konvektiv-dispersiver Modelle zur Berechnung des instationären Stofftransports in ungestörten Bodensäulen. Ph.D. Dissertation. University of Bayreuth, Germany. 


\section{Figures}

Fig. 1. Flow patterns observed in a field experiment at the three sites Les Barges, Bülach, and Wülflingen after infiltration of $40 \mathrm{~mm}$ of a dye tracer solution. The square plots on the left-hand side show the dye coverage digitized from photographs of the vertical soil profiles. The depth profiles in the middle are percentages of dye coverage averaged per depth. The transport processes in field soils may range from homogeneous matrix flow to fingering and macropore flow. Different flow regimes may occur combined within the same soil (e.g., at the site Wülflingen). (Figure adapted from Flury et al., 1994.)

Fig. 2. Schematic of three basically different regimes of lateral water and solute mass exchange: Solutes separate into preferential ports (attractor zone), percolate in isolated flow regions (transmission zone), and disperse into the matrix (dispersion zone).

Fig. 3. A soil may be considered as a composite of parallel flow regions of differing velocities. The travel distance $\mathbf{z}$ after time $t=n \Delta t$ is the sum of $n$ small travel distances $z_{i}$. Depending on the scale of observation such flow regions may represent single pores, macrostructural channels or otherwise preferentially wetted regions extending more or less in the main direction of flow.

Fig. 4. Effect of the flow regime on the vertical distribution of travel distances of particles. Depending on the heterogeneity of the flow field the distribution of the local velocity $v$ may exhibit any shape. Here in this hypothetical example, $v$ is bimodal log-normally distributed (Eq. 9). The graphs for $f(z)$ were calculated with (2), (7), and (8) for two different times $t_{1}<t_{2}$. The dashed line indicates the mean of the distributions and the shaded area covers one standard deviation. 

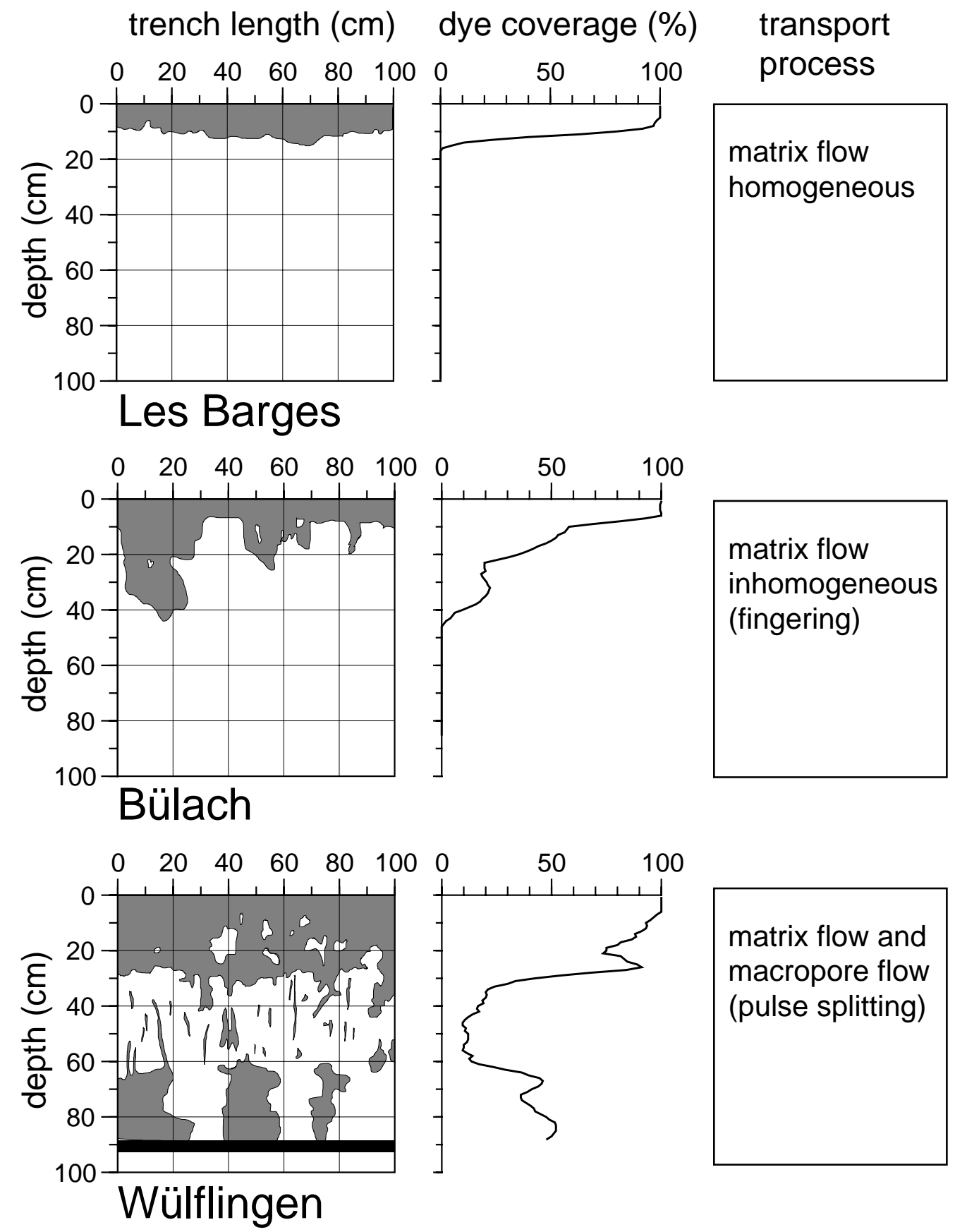

Fig. 1

Flühler et al. : Lateral Mixing Processes-A Key for Understanding the Solute Transport Regime Fig. 1. 

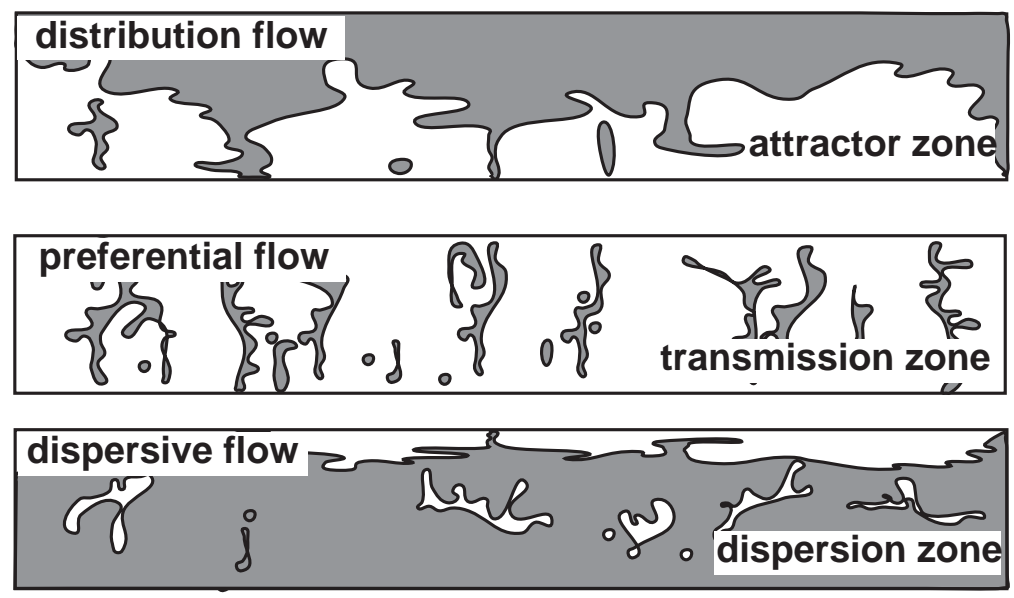

Fig. 2

Flühler et al.: Lateral Mixing Processes-A Key for Understanding the Solute Transport Regime

Fig. 2. 


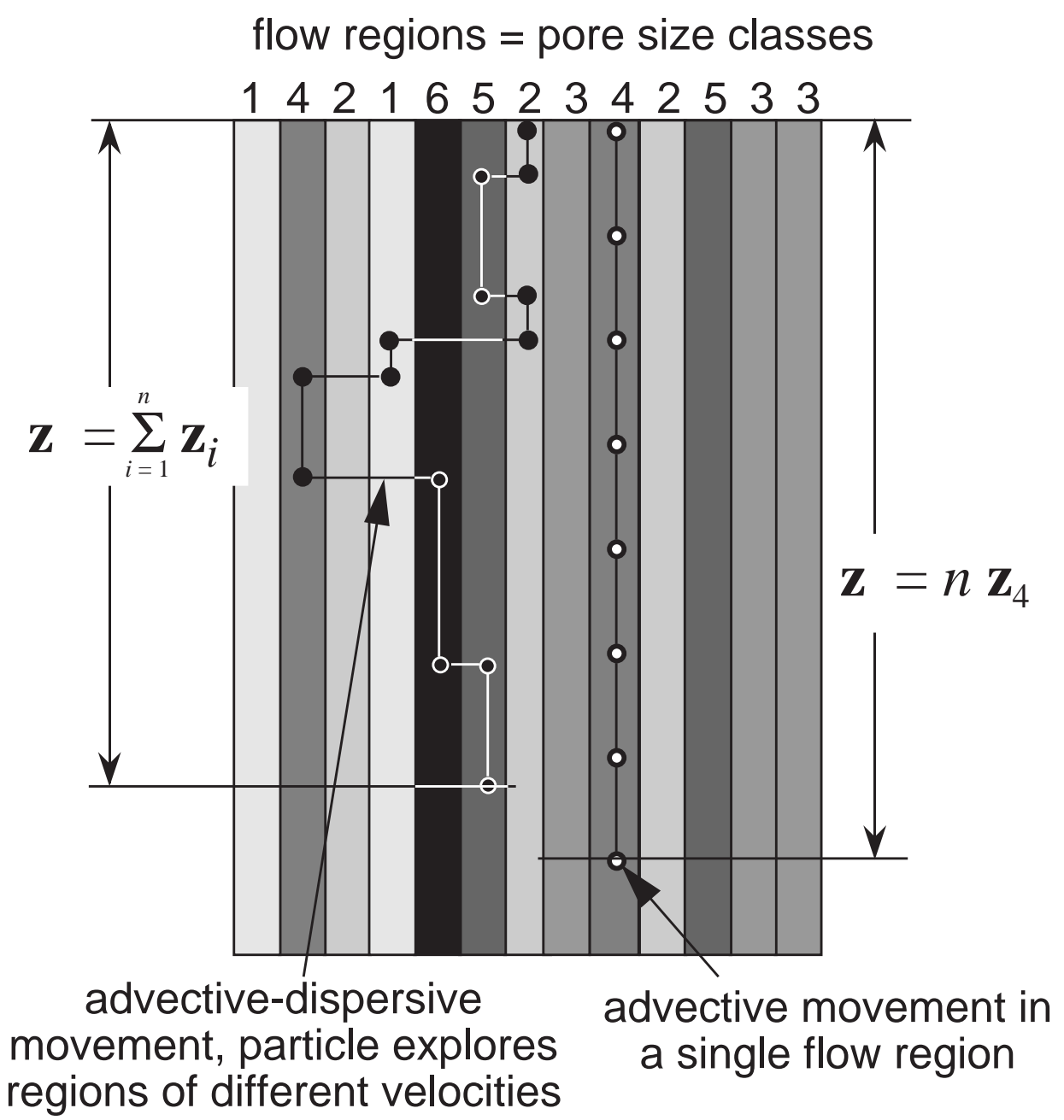

Fig. 3

Flühler et al.: Lateral Mixing Processes-A Key for Understanding the Solute Transport Regime

Fig. 3. 


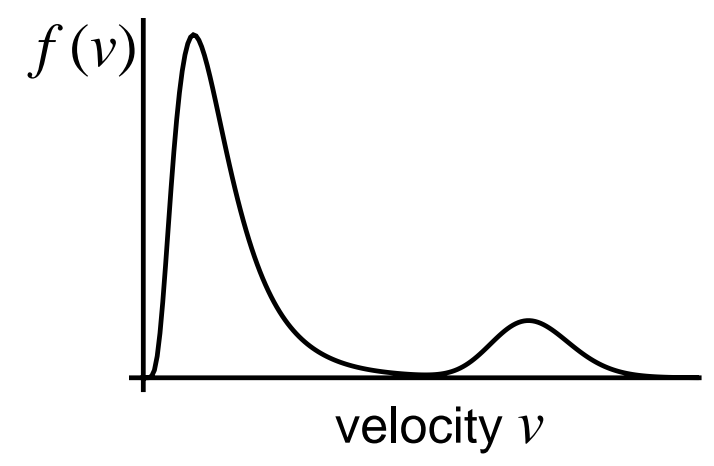

stochastic-advective advective-dispersive
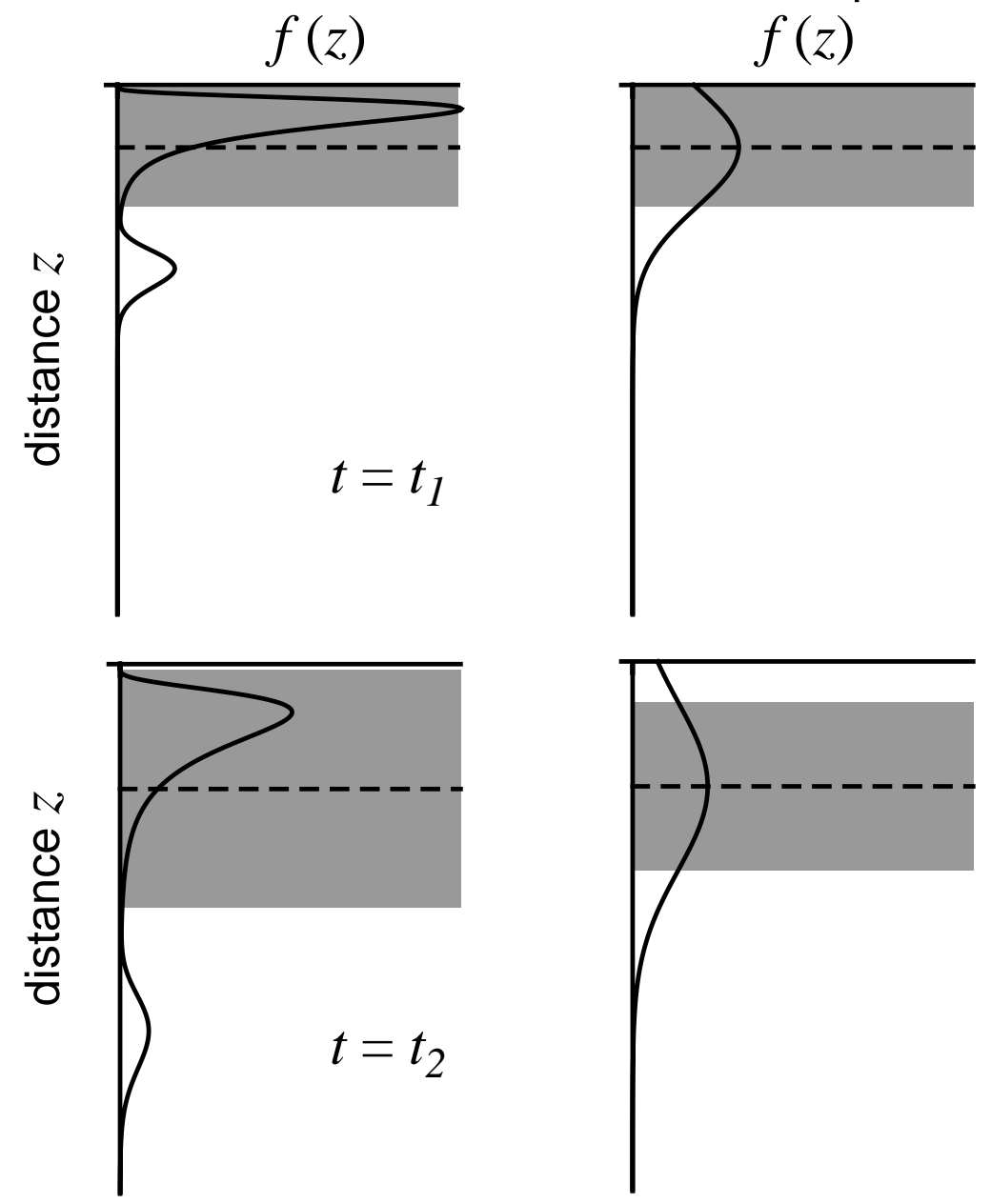

Fig. 4

Flühler et al. : Lateral Mixing Processes-A Key for Understanding the Solute Transport Regime Fig. 4. 